\title{
Conhecimento, atitude e prática de mulheres residentes no meio rural acerca dos métodos contraceptivos
}

\author{
The knowledge, attitudes and practices of women living in rural environments regarding contraceptive methods
}

Conocimiento, actitud y práctica de mujeres residentes en medio rural acerca de métodos anticonceptivos

Ana Izabel Oliveira Nicolau', Renata Cordeiro Dantas², Ana Paula Pires Gadelha ${ }^{3}$, Ana Karina Bezerra Pinheiro ${ }^{4}$

\section{RESUMO}

Estudo descritivo-quantitativo com objetivo de verificar o conhecimento, a atitude e a prática de mulheres residentes em uma comunidade rural quanto a métodos contraceptivos. Foi aplicado, nessa perspectiva o Inquérito Conhecimento, Atitude e Prática (CAP) a 50 usuárias do serviço de planejamento familiar da unidade básica de saúde de Pedro Ribeiro de Russas, - CE, em julho de 2008. Foi detectada a predominância de mulheres jovens, unidas com seus parceiros e com baixa escolaridade e renda. Também, observaram-se antecedentes obstétricos em 46 (92\%) mulheres, abortos em 13 (26\%) e 18 (36\%) com história de gravidez indesejada. O anticoncepcional oral, a camisinha, a tabela de Ogino-Knaus e o Dispositivo Intrauterino (DIU) foram pouco empregados. Salienta-se que 14 (28\%) utilizavam algum método de maneira inadequada. As principais fontes de orientação foram o profissional de saúde e amigos. As características culturais, sociodemográficas e as circunstâncias vivenciadas influenciaram o conhecimento, atitude e uso de métodos contraceptivos e, consequentemente, a sua história reprodutiva.

Descritores: Planejamento familiar; População Rural; Saúde da Mulher; Enfermagem.

\section{ABSTRACT}

This descriptive, quantitative study was performed with the objective to verify the knowledge, attitudes and practices of women living in a rural community regarding contraceptive methods. The Knowledge, Attitude and Practice Survey was applied to 50 clients of the family planning service of the Pedro Ribeiro de Russas (CE) Basic Health Unit, in July 2008. Most women were young, living with their partners and had a low level of education and income. Of all the women, 46 (92\%) had an obstetrical history, 13 (26\%) had had abortions/miscarriages, and 18 (36\%) had a history of unwanted pregnancy. Oral contraception, condoms, the Ogino-Knaus table and the Intra-Uterine Device (IUD) were infrequently used. Fourteen (28\%) women used some method of birth control, but used it inadequately. Their main sources of guidance were health professionals and friends. Their cultural and sociodemographic characteristics and their life circumstances affected their knowledge, attitudes and practices regarding contraceptive methods and, consequently, their reproductive history.

Descriptors: Family Planning; Rural Population; Women's Health; Nursing.

\section{RESUMEN}

Estudio descriptivo cuantitativo que objetiva verificar conocimiento, actitud y práctica de mujeres residentes en una comunidad rural sobre métodos anticonceptivos. Se aplicó el Cuestionario Conocimiento, Actitud y Práctica (CAP) con 50 pacientes del servicio de planificación familiar de unidad básica de salud de Pedro Ribeiro de Russas-CE, en julio 2008. Predominancia de mujeres jóvenes, en pareja, con baja renta y escolaridad. Antecedentes obstétricos en 46 (92\%) mujeres, abortos en 13 (26\%) y 18 (36\%) pacientes con historia de embarazo no deseado. El anticonceptivo oral, el preservativo, la tabla de Ogino-Knaus y el Dispositivo Intra-Uterino (DIU) fueron poco utilizados. Se destaca que 14 (28\%) utilizaron algún método inadecuadamente. Las principales fuentes de orientación fueron profesionales de salud y amistades. Las características culturales, sociodemográficas y las circunstancias experimentadas influyeron en el conocimiento, actitud y práctica de métodos anticonceptivos y, consecuentemente, en la historia reproductiva.

Descriptores: Planificación Familiar; Población Rural; Salud de la Mujer; Enfermería.

\footnotetext{
${ }^{1}$ Enfermeira, Mestre em Enfermagem, Discente do Programa de Pós-Graduação em Enfermagem - nível Doutorado, Universidade Federal do Ceará (UFC). Picos, Pl, Brasil. E-mail: anabelpet@yahoo.com.br.

2 Enfermeira. Fortaleza, CE, Brasil. E-mail: renatacdantas@gmail.com.

${ }^{3}$ Enfermeira. Fortaleza, CE, Brasil. E-mail: anapaula_enfe@hotmail.com.

${ }^{4}$ Enfermeira, Doutora em Enfermagem, Professora Adjunto III, UFC. Fortaleza, CE, Brasil. E-mail: anakarinaufc@hotmail.com.
} 


\section{INTRODUÇÃO}

No Brasil, ao longo do século $\mathrm{XX}$, ocorreu uma melhoria significativa da atenção pública à saúde reprodutiva através da construção de diferentes práticas e conhecimentos especializados, os quais começaram a atender as necessidades sociais, os problemas e carências relacionados à reprodução humana.

No início dos anos 80, o Ministério da Saúde criou o Programa de Atenção Integral à Saúde da Mulher (PAISM), sendo considerado um marco histórico das políticas de gênero no país. Tal programa inseriu uma nova abordagem à saúde da mulher, que incluiu dentre suas ações, questões relativas ao planejamento familiar, adotando políticas e medidas para permitir o acesso da população aos meios de contracepção(1).

O planejamento familiar, a exemplo de tantas outras problemáticas de ordem pública, é sempre um assunto atual e permanentemente debatido. Entretanto, causam inúmeras controvérsias nos mais diversos setores da sociedade, de modo que há segmentos que concordam com o planejamento familiar, pelo fato deste incorporar interesses político-estratégicos de controle de parcelas populacionais específicos.

A anticoncepção tem adquirido papel importante na saúde reprodutiva e seu uso de forma inadequada implica agravos à saúde da mulher, como gravidez indesejada, gravidez na adolescência, abortamentos ilegais e até mesmo aumento na mortalidade materna. Realizar planejamento familiar reduz a morbidade e a mortalidade materno-infantil resultantes, principalmente, de abortamento provocado ou de gestação de alto risco(2).

A liberdade de escolha é fundamental na área do controle da fecundidade. Para que os casais possam optar por um método contraceptivo de forma livre e informada, precisam conhecer e ter acesso a todos os métodos anticoncepcionais cientificamente aprovados e disponíveis, escolhendo aquele que seja mais adequado às suas características e suas condições de vida em cada momento. Dentro dessa proposta, o planejamento familiar tem como objetivo garantir às mulheres e aos homens um direito básico à cidadania: o direito de ter ou não filhos ${ }^{(3)}$.

A implementação dos serviços de planejamento familiar precisam de constante avaliação e aprimoramento, de modo a considerar as características epidemiológicas e culturais da comunidade adscrita, para que sua finalidade seja congruente com as necessidades dos casais. Ademais, é premente considerar os conhecimentos e atitudes (opiniões) no processo de desenvolvimento de habilidades em contracepção. Diante do exposto questiona-se qual o conhecimento, a atitude e a prática de mulheres residentes no meio rural, acerca dos métodos contraceptivos?

Acredita-se que os resultados do estudo poderão subsidiar a elaboração de ações educativas de cunho grupal, bem como individual, que visem aprimorar o conhecimento das usuárias do serviço de planejamento familiar de acordo com suas reais carências. Conhecendo e entendendo como funcionam esses métodos, as usuárias e seus parceiros terão a oportunidade de escolher qual se adéqua melhor às suas necessidades, levando em consideração aspectos biológicos, sociais, econômicos, culturais e religiosos. Trata-se do fortalecimento dos direitos sexuais e reprodutivos, para que não haja uma escolha contraceptiva precipitada sem conhecimento, experiência e clareza necessária.

Os resultados indicarão uma conjuntura diagnóstica sobre o conhecimento, a atitude e a prática do grupo feminino estudado, cuja avaliação poderá contribuir para a reorientação das condutas profissionais ora adotadas, uma vez que aperfeiçoará a visão dos profissionais de saúde. Ressalta-se que o enfermeiro, como membro da equipe da Estratégia Saúde da Família (ESF), é responsável direto pela implementação dos serviços de saúde sexual e reprodutiva, através de uma abordagem integral com vistas à promoção da saúde da comunidade.

Ademais, os resultados evidenciarão o contexto sócio-demográfico e reprodutivo das mulheres residentes em meio rural, revelando lacunas e riscos que conduzirão estratégias para a promoção da saúde dessa clientela. Portanto, o estudo em questão pretende verificar o conhecimento, a atitude e a prática de mulheres residentes no meio rural acerca dos métodos contraceptivos.

\section{METODOLOGIA}

Trata-se de um estudo avaliativo do tipo Conhecimento, Atitude e Prática (CAP) de corte transversal e abordagem quantitativa. Os estudos CAP pertencem a uma categoria de estudos avaliativos, chamados de avaliação formativa, ou seja, para além de se obter dados de uma parcela populacional específica, estes identificam possíveis caminhos para uma futura 
intervenção mais eficaz. Portanto, o estudo CAP consiste em um conjunto de questões que visam medir o que a população sabe, pensa e atua frente a um tema prédefinido(4) $^{(4)}$

A coleta de dados foi realizada na comunidade rural Pedro Ribeiro, no município de Russas, interior do estado do Ceará, em julho de 2008. A amostra foi composta por mulheres que obedeceram aos seguintes critérios de inclusão: estar na faixa etária entre 18 e 50 anos, ter vida sexual ativa, utilizar algum método contraceptivo e ser atendidas no serviço de planejamento familiar da unidade básica de saúde local, contabilizando 50 mulheres. Ressalta-se que, no total, 70 mulheres realizam o acompanhamento na unidade de saúde sede do estudo, porém 20 enquadraram-se nos critérios de exclusão.

As mulheres que aguardavam atendimento ginecológico e de planejamento familiar foram convidadas a contribuir com o estudo. Antes do início da coleta de dados foi requerida a autorização das participantes, após o esclarecimento dos objetivos do estudo e dos aspectos éticos, mediante a assinatura do Termo de Consentimento Livre e Esclarecido. Os dados foram coletados na Unidade de Saúde da Família da referida comunidade, utilizando-se de um formulário estruturado do tipo Inquérito Conhecimento, Atitude e Prática (CAP) sobre o uso de métodos contraceptivos.

A técnica de entrevista foi aplicada com cada participante em um espaço reservado dentro na unidade de saúde. O instrumento de coleta primeiramente contemplou dados de caracterização sociodemográfica e história obstétrica, seguidas das informações sobre a prática e o conhecimento dos métodos contraceptivos (método empregado, tempo de uso e descrição do uso) e finalizou com as informações de atitude frente à contracepção (fonte de informação sobre os métodos e necessidade do serviço de planejamento familiar nas escolhas contraceptivas).

A análise dos dados baseou-se na descrição em forma de gráficos e tabelas ilustrativos, com frequências absolutas e relativas, sendo posteriormente analisados de acordo com a literatura pertinente.

Os aspectos éticos da pesquisa com seres humanos foram respeitados, segundo a Resolução n 196, de 1996 do Conselho Nacional de Saúde ${ }^{(5)}$. O estudo foi realizado após aprovação do projeto de pesquisa pelo Comitê de
Ética em Pesquisa da Universidade Federal do Ceará (UFC), sob protocolo n 108/08.

\section{RESULTADOS E DISCUSSÃO}

Os resultados de identificação sociodemográfica de 50 mulheres residentes na comunidade rural Pedro Ribeiro evidenciaram uma população feminina jovem com 31 mulheres (62\%) pertencentes à faixa etária entre 18 e 30 anos e unida maritalmente em 34 (68\%). A baixa escolaridade e renda foram evidentes de modo que 37 (74\%) possuíam no máximo o ensino fundamental e 46 (92\%) possuíam rendimento familiar de até $\mathrm{R} \$ 500,00$, variando de nenhuma renda até o valor anteriormente referido. Quanto à ocupação laboral, a principal foi voltada para a agricultura em 23 (46\%), seguida do trabalho como empregada doméstica por nove (18\%) mulheres.

Tais achados ratificam a importância do fortalecimento de estratégias em planejamento familiar nas comunidades rurais para que haja uma escolha contraceptiva adequada e consciente dos aspectos envolvidos em suas decisões contraceptivas. Trata-se de uma população carente de uma assistência em planejamento familiar, que ressalte não somente à solução dos problemas relativos ao controle da prole, mas que enfatize a adoção de atitudes saudáveis para a garantia de uma vida sexual segura.

O processo de escolha informada na regulação da fecundidade baseia-se nos princípios de proporcionar bem-estar às pessoas, quanto à sua autonomia, expectativas, necessidades e poder de decisão, enfocando especialmente os direitos sexuais e reprodutivos, na qualidade de direitos humanos individuais(6).

O perfil sociodemográfico relaciona-se ao futuro reprodutivo da população. Pesquisa realizada em âmbito nacional evidenciou que as mulheres com fecundidade mais elevada eram aquelas com rendimento domiciliar mensal per capita de até $1 / 4$ de salário mínimo e com até três anos de estudo ${ }^{(7)}$.

Em razão da gravidez muitas adolescentes abandonam a escola e poucas retornam. Esta realidade pode estar relacionada à baixa escolaridade e continuidade dos estudos entre as mulheres do meio rural, uma vez que estas abandonam a escola mais precocemente para assumir papéis, como o de esposa, dona de casa, mãe, entre outros. Salienta-se que apenas 
quatro (8\%) mulheres da amostra estudada ainda frequentam a escola refletindo as dificuldades para o prosseguimento das atividades escolares.

Apesar das dificuldades a taxa de fecundidade caiu em todo o país, até mesmo nas regiões mais pobres, como é o caso da população pobre, rural, de menor escolaridade, negra e/ou residente no Nordeste, ainda que de forma relativamente mais lenta, quando comparado ao que ocorreu nas décadas anteriores e ao que vem ocorrendo com o restante da população(7).
Destaca-se que a inserção da mulher no mercado de trabalho vem contribuindo no declínio da natalidade ocorrido no Brasil nos últimos $30 \operatorname{anos}^{(8)}$. Observa-se que, a atual competição vem despertando novos interesses, pela ascensão social, pela necessidade de sobrevivência, surgindo daí a preocupação em diminuir a fecundidade, e consequentemente a necessidade de um planejamento familiar adequado.

Os dados referentes à história obstétrica das mulheres participantes do estudo foram dispostos no Gráfico 1:

Gráfico 1: Antecedentes obstétricos de mulheres residentes em uma comunidade rural. Pedro Ribeiro, Russas-CE, julho de 2008.

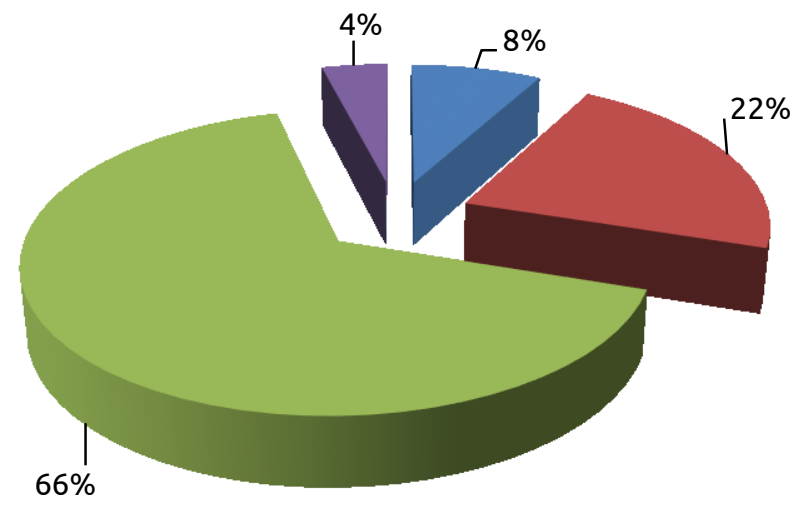

- Sem antecedentes obstétricos

- História de aborto e parto

- História de parto

- História de aborto
A distribuição dos dados destaca uma realidade em que apenas quatro (8\%) das mulheres que realizavam planejamento familiar não possuíam antecedentes obstétricos. Esse achado nos remete à reflexão de como estão sendo realizadas estratégias de cunho preventivo da gravidez indesejada, especialmente durante a adolescência.

Historicamente, a população feminina vem gestando durante a fase da adolescência, mesmo em um contexto de intensa redução da fecundidade, não se observou no Brasil um deslocamento da reprodução para faixas etárias mais velhas ${ }^{(9)}$. Portanto, é de suma importância a disseminação de informações sobre planejamento familiar e promoção da saúde sexual nos espaços ocupados por essa população, especialmente nas escolas, para que não sejam surpreendidos por situações indesejáveis devido à falta de esclarecimento e orientação.

Porém a carência das ações em planejamento familiar na comunidade rural foi ratificada pela alta frequência 13 (26\%) de abortos. O abortamento induzido pela mulher é considerado um problema de Saúde Pública, visto que está associado ao alto índice de mortalidade, sendo a quarta causa de morte materna, devido a complicações. Além disso, envolve questões éticas, morais, religiosas e emocionais ${ }^{(10)}$.

Quando indagadas sobre a ocorrência de gravidez indesejada, 18 (36\%) responderam que tiveram gestações que não estavam dentro do seu planejamento familiar. A gravidez não planejada é duas vezes mais comum entre as mulheres que vivem em áreas onde os serviços de planejamento familiar são de baixa qualidade ${ }^{(11)}$. Percebe-se a necessidade de programas de atenção à saúde sexual e reprodutiva específicas às mulheres residentes em comunidades rurais com vistas a prevenir gravidezes indesejadas e diminuir a incidência de abortos provocados ou espontâneos.

Apesar dos inúmeros métodos anticoncepcionais existentes, as mulheres da comunidade Pedro Ribeiro utilizam apenas quatro tipos de acordo com a seguinte distribuição (Gráfico 2). 
Gráfico 2: Distribuição contraceptiva de mulheres residentes em uma comunidade rural. Pedro Ribeiro, Russas-CE, julho de 2008.

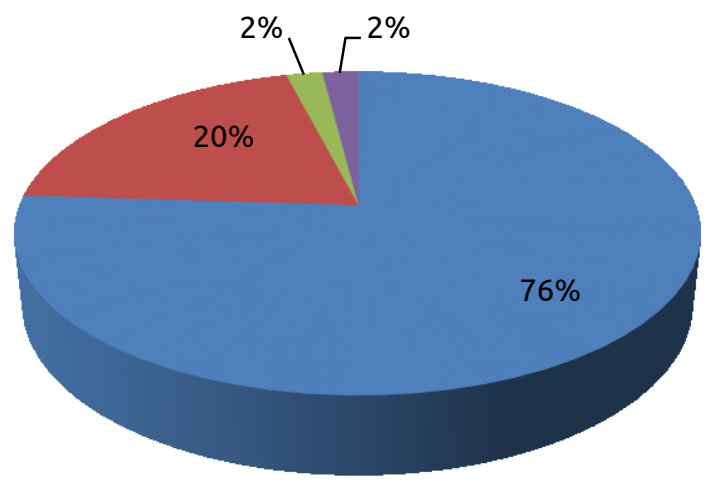

Anticoncepcional oral

- Preservativo masculino

- Tabela de Ogino-Knaus

- Dispositivo Intra-uterino (DIU)
No caso deste estudo, a forte concentração na pílula pode ser atribuída, em grande parte, a carência de um programa de planejamento familiar adequado, em que sejam fornecidas informações suficientes ao cliente sobre outros métodos existentes e sua disponibilidade. Ademais, como se trata de uma população de baixo poder aquisitivo, caso os métodos não sejam oferecidos gratuitamente, os casais, muitas vezes, não têm condições de adquiri-los.

A escolha pela pílula, na maioria das vezes, é precipitada e desconsidera seus efeitos colaterais e contra-indicações. É um método disseminado na população feminina, chegando a ser utilizada de forma empírica e sem indicação por um profissional de saúde.

Tal realidade foi ratificada por estudo realizado com 284 mulheres contempladas pela Estratégia Saúde da Família da cidade de Maringá-PR em que constatou que apenas um terço das usuárias de anticoncepcional oral se submete previamente a consulta médica, além disso, a prevalência do uso ocorre em faixas etárias mais jovens, geralmente antes dos 30 anos $^{(2)}$. Portanto, corresponde a faixa etária em que há maior variedade de parceiros sexuais e maiores chances de engravidar, fatores que denotam a grande preocupação pela decisão contraceptiva, contrapondo-se a vulnerabilidade de aquisição de DST/aids.

Quanto à utilização de métodos de barreira, a camisinha masculina apareceu como o segundo método contraceptivo mais utilizado por 10 (20\%) participantes. Achado positivo, uma vez que, apesar de estar sendo utilizado рага o fim anticoncepcional confere concomitante proteção às DST/aids. Estudo realizado na cidade de Belo Horizonte-MG com o intuito de acompanhar a implementação de grupos de planejamento familiar, demonstrou que as mulheres que utilizam a camisinha masculina como método contraceptivo associam a esta também a finalidade de proteção às DST/aids, já que a infidelidade é citada como integrante da "natureza" do homem"(12).

Dentre os atributos negativos relacionados ao uso do preservativo, os mais apontados são a interferência no prazer e a necessidade de interrupção dos jogos amorosos para a colocação da camisinha ${ }^{(12)}$.

Notou-se a ausência de associação entre o uso do anticoncepcional oral e a camisinha. Fato confirmado em outro estudo realizado na capital cearense, em que a utilização do contraceptivo hormonal foi o fator mais interferente para a não aceitação do preservativo por parte do parceiro ${ }^{(13)}$. Portanto, nota-se uma exclusão da preocupação com a vulnerabilidade às DST/aids, prevalecendo o interesse pela decisão contraceptiva.

No concernente aos métodos comportamentais de planejamento familiar, segundo o Ministério da Saúde são técnicas para obter ou evitar a gravidez mediante a observação de alterações que ocorrem no organismo feminino durante o ciclo menstrual. Além disso, existem práticas sexuais, a relação sexual sem penetração e a interrompida antes da ejaculação (coito interrompido), que são consideradas métodos comportamentais, já que reduzem o risco de uma gravidez indesejada ${ }^{(14)}$.

No presente estudo, o único método comportamental mencionado foi a tabela de Ogino Knaus por uma (2\%) mulher. Pesquisa realizada na cidade do Rio de Janeiro apontou que os métodos naturais ainda são os menos conhecidos e utilizados ${ }^{(15)}$.

Dentre as causas para a descontinuidade/falha dos métodos comportamentais estão a necessidade da abstinência sexual por vários dias do ciclo menstrual, 
baixa confiabilidade, aliado à falta de conhecimento feminina sobre seu ciclo menstrual e período fértil.

No referente ao uso do dispositivo intra-uterino (DIU), esse foi citado por uma (2\%) mulher, retratando sua baixa aceitação, muitas vezes ocasionada pela falta de informação e o temor pelos possíveis efeitos colaterais, principalmente aumento das cólicas e sangramento irregular/severo. Além disso, das gestações ocorridas em usuárias do DIU, 3 a 5\% são ectópicas ${ }^{(14)}$.

Apesar do baixo custo de um DIU, é notória a sua baixa prevalência, possivelmente devido à falta do insumo, deficiência de capacitação profissional na técnica de inserção, carência de orientações acerca do método, mitos de usuárias e profissionais ou uma conjunção de todas as causas(16).

Os serviços de saúde devem garantir o acesso aos meios para evitar gravidez, além de consultas ginecológicas e ações educativas para a escolha consciente do método(16). Ademais, diante das opções oferecidas para a escolha do contraceptivo, é sempre importante que os casais participem de programas de planejamento familiar para que, com o apoio dos profissionais de saúde, possam fazer suas decisões de maneira mais adequada.

Quanto ao tempo de uso dos métodos contraceptivos, 28 (56\%) mulheres usavam há mais de 12 meses, 11 (22\%) entre três e sete meses, sete (14\%) entre oito a 12 meses e quatro (8\%) há menos de três meses. A efetividade contraceptiva é um dos aspectos mais importantes na escolha do método, sendo avaliada pelo número de gestações não-planejadas que ocorrem durante um período de exposição específico na vigência de um determinado método contraceptivo ${ }^{(18)}$.

Ressalta-se que a pílula combinada, o método mais empregado no presente estudo, consiste em um método de média eficácia no uso rotineiro e de alta eficácia quando usada de forma correta e consistentemente. A eficácia do método, para cada caso individual, dependerá fundamentalmente da maneira como ele é utilizado(19).

Ao se investigar o conhecimento da prática contraceptiva entre as mulheres participantes, 36 (72\%) utilizavam o método escolhido de maneira correta, enquanto 14 (28\%) não faziam o uso adequadamente. 0 desconhecimento, a insegurança, a existência de mitos favorecem interpretações errôneas frente às falhas dos métodos e efeitos colaterais ${ }^{(3)}$.
O problema do uso inadequado, especialmente o da pílula, e a ausência de acompanhamento pela equipe de saúde agravam-se quando se verifica que 28 (56\%) usuárias do método estão o utilizando por mais de um ano.

Os achados supracitados denotam as dificuldades do planejamento familiar no Brasil no seguimento dos seis elementos fundamentais para a qualidade de atenção em planejamento familiar, a saber: escolha livre de métodos, informação para usuárias, competência técnica, relação usuária-serviço, acompanhamento e integração do planejamento familiar ao atendimento em saúde reprodutiva ${ }^{(6)}$.

Nos dados referentes à fonte de informação 28 (56\%) mulheres foram orientadas quanto ao uso do método contraceptivo escolhido pelo profissional de saúde, 18 (36\%) foram orientadas por amigos, uma (2\%) usuária foi orientada através dos meios de comunicação, duas (4\%) iniciaram o uso por conta própria, e ainda uma (2\%) foi orientada pelo balconista da farmácia.

Algumas mulheres controlam a sua fecundidade sem a assistência de um profissional de saúde, não sentindo necessidade de serviços educacionais ou de métodos prescritos, ficando mais suscetíveis a possíveis transtornos, falhas e efeitos colaterais.

Apesar de todas as 50 mulheres participarem de programas de planejamento familiar, nem todas atribuem a sua devida importância, visto que 10 (20\%) participantes não consideram este serviço importante para utilização e escolha do método.

O programa de ação da Conferência Internacional sobre População e Desenvolvimento (ICPD) que teve lugar no Cairo em 1994, reconheceu a importância dos governos concordarem em redobrar os esforços para a implementação de Serviços e Programas de Planejamento Familiar e Saúde Reprodutiva. Porém é preciso reafirmar os preceitos no planejamento familiar no Brasil para que realmente sejam garantidas livres escolhas contraceptivas, informações aos casais, competência técnica, acompanhamento e integração do planejamento familiar ao atendimento em saúde reprodutiva(6). Mediante tais melhorias, a valorização e reconhecimento dessa iniciativa seriam percebidos de modo mais espontâneo pelos casais na condução de suas histórias reprodutivas. 


\section{CONCLUSÕES}

As características culturais e sociodemográficas, bem como as circunstâncias vivenciadas pelas mulheres da comunidade rural de Pedro Ribeiro, influenciaram o conhecimento, atitude e prática dos métodos contraceptivos e, consequentemente, a sua história reprodutiva. Uma das medidas para melhorar os problemas relativos à saúde reprodutiva seria o fortalecimento da Estratégia Saúde da Família (ESF) no que tange ao acesso à saúde em geral e boa acessibilidade ao planejamento familiar, abrangendo os contextos sócio-familiares das mulheres residentes na zona rural.

\section{REFERÊNCIAS}

1. Osis MJD, Faúndes A, Makuch MY, Mello MB, Sousa MH, Araújo MJO. Atenção ao planejamento familiar no Brasil hoje: reflexões sobre os resultados de uma pesquisa. Cad. Saúde Pública. 2006;22(11):2481-90.

2. Souza JMM, Pelloso SM, Uchimura NS, Souza F. Utilização de métodos contraceptivos entre as usuárias da rede pública de saúde do município de Maringá-PR. Rev. Bras. Ginecol. Obstet. 2006;28(5):271-7.

3. Andrade EC, Silva LR. Planejamento familiar: uma questão de escolha.Rev. Eletr. Enf. [Internet]. 2009 [cited 2012 mar 30]; 11(1):85-93. Available from:

http://www.fen.ufg.br/revista/v11/n1/v11n1a11.htm

4. Ministério da Educação. Instituto Nacional do Desenvolvimento da Educação. Manual do aplicador do estudo CAP [Internet]. 2002 [cited 2008 out 09]. Available from: http://www.inde.gov.mz/docs/monieduca10.doc

5. Conselho Nacional de Saúde. Resolução n. 196, de 10 de outubro de 1996. Dispõe sobre diretrizes e normas regulamentadoras de pesquisas envolvendo seres humanos. Bioética. 1996; 4(Supl 2):1525.

6. Osis MJD, Duarte GA, Crespo ER, Espejo X, Pádua KS. Escolha de métodos contraceptivos entre usuárias de um serviço público de saúde. Cad. Saúde Pública. 2004;20(6):1586-94.

7. Pesquisa Nacional sobre Demografia e Saúde. PNDS-2006: cai a taxa de fecundidade no Nordeste. Brasília, DF: Agencia

Saúde/Demografia e Saúde [Internet]. 2008 [cited 2008 set 20].

Available from:

http://portal.saude.gov.br/portal/arquivos/pdf/pndsnordeste.pdf

8. Coelho EBS. Enfermagem e o planejamento familiar: as interfaces da contracepção. Rev. Bras. Enferm. 2005;58(6):665-72.

9. Aquino PS. Desempenho das atividades de vida por prostitutas de Fortaleza [dissertation]. Fortaleza: Faculdade de Farmácia,

Odontologia e Enfermagem/UFC; 2007.

10. Matos JC, Luz GS, Pelloso SM, Carvalho MDB. Mortalidade por aborto no Estado do Paraná: 1998 a 2004. Rev. Eletr. Enf. [Internet]. 2007 [cited 2012 mar 30]; 9(3):806-14. Available from:

http://www.fen.ufg.br/revista/v9/n3/v9n3a19.htm.

11. Population Reports. Programas de planejamento familiar: melhoria de qualidade. Population Information Program. Maryland, USA [Internet]. 2008 [cited 2008 nov 13]. Available from:

http://bibliomed.uol.com.br/lib/ShowDoc.cfm?LibDoclD=12040\&Ret urnCatID=503

12. Moreira MHC, Araújo JNG. Planejamento familiar: autonomia ou encargo feminino? Psicologia em Estudo. 2004;9(3):389-98.

13. Carvalho ALS, Bezerra SJS, Leitão NMA, Joca MT, Pinheiro AKB. Porte, acondicionamento e utilização de preservativo masculino entre jovens de Fortaleza - um estudo descritivo. Online braz. j. nurs. [Internet]. 2006 [cited 2008 out 16]; 6(0). Available from:
A motivação para a participação dos serviços de planejamento familiar e uso dos métodos contraceptivos deve ser estimulada considerando as condições sócioculturais, necessidades de cada casal, escolhas contraceptivas respaldas pela informação e orientação por profissionais de saúde, e disponibilidade dos métodos, para que os casais não sejam reconduzidos em suas escolhas pela ausência do método desejado. Ressalta-se que os profissionais de saúde precisam aprimorar sua capacidade de comunicação com os usuários, uma vez que esta assume um papel primordial na garantia de escolhas informadas a fim de emponderar a população na tomada de suas decisões em saúde.

http://www.uff.br/objnursing/index.php/nursing/article/view/566, 2 007.

14. Ministério da Saúde. Assistência em Planejamento Familiar. Série A. Normas e manuais técnicos. n.40. Brasília (Brasil): Ministério da Saúde; 2002.

15. Andrade EC, Silva LR. Planejamento familiar: uma questão de escolha. Rev. Eletr. Enf. [Internet]. 2009 [cited 2012 mar 30];11(1):85-93. Available from:

http://www.fen.ufg.br/revista/v11/n1/v11n1a11.htm.

16. Bahamondes L. A escolha do método contraceptivo. Rev. Bras. Ginecol. Obstet. 2006;28(5):267-70.

17. Nicolau AIO, Aquino OS, Pinheiro AKP. Caracterização social de prostitutas diante da visão integral da saúde. Rev. Min. Enferm. 2008;12(1):11-6.

18. Duncan BB, Schmidht MI, Giugliani ERJ. Medicina ambulatorial: condutas de atenção primária baseadas em evidências. $3^{\mathrm{a}}$ ed. Porto Alegre: Artmed; 2004.

19. Díaz J, Chinaglia M, Díaz M, Bossemeyer R, Marinho A, Santos L. Manual de anticoncepção on-line. Anticoncepção on-line [Internet]. 2006 [cited 2006 out 25]. Available from: http://www.anticoncepcao.org.br/html/manual/manual.htm.

Artigo recebido em 02.08.2010.

Aprovado para publicação em 13.03.2012.

Artigo publicado em 30.03.2012. 